\title{
Association between delayed bedtime and sleep-related problems among community- dwelling 2-year-old children in Japan
}

Shingo Kitamura ${ }^{1}$, Minori Enomoto ${ }^{1}$, Yuichi Kamei ${ }^{1}$, Naoko Inada ${ }^{2}$, Aiko Moriwaki ${ }^{3}$, Yoko Kamio ${ }^{4}$ and Kazuo Mishima ${ }^{1 *}$

\begin{abstract}
Background: Although delayed sleep timing causes many socio-psycho-biological problems such as sleep loss, excessive daytime sleepiness, obesity, and impaired daytime neurocognitive performance in adults, there are insufficient data showing the clinical significance of a 'night owl lifestyle' in early life. This study examined the association between habitual delayed bedtime and sleep-related problems among community-dwelling 2-year-old children in Japan.

Methods: Parents/caregivers of 708 community-dwelling 2-year-old children in Nishitokyo City, Tokyo, participated in the study. The participants answered a questionnaire to evaluate their child's sleep habits and sleep-related problems for the past 1 month.

Results: Of the 425 children for whom complete data were collected, 90 (21.2\%) went to bed at 22:00 or later. Children with delayed bedtime showed significantly more irregular bedtime, delayed wake time, shorter total sleep time, and difficulty in initiating and terminating sleep. Although this relationship indicated the presence of sleep debt in children with delayed bedtime, sleep onset latency did not differ between children with earlier bedtime and those with delayed bedtime. Rather, delayed bedtime was significantly associated with bedtime resistance and problems in the morning even when adjusting for nighttime and daytime sleep time.

Conclusions: Even in 2-year-old children, delayed bedtime was associated with various sleep-related problems. The causal factors may include diminished homeostatic sleep drive due to prolonged daytime nap as well as diurnal preference (morning or night type) regulated by the biological clock.
\end{abstract}

Keywords: Sleep habits, Sleep problems, Toddlers, Prevalence, Cross-sectional study

\section{Background}

Approximately $25 \%$ of children are reported to experience some type of sleep problem [1]. The most commonly reported sleep-related problems are sleep loss and subsequent daytime dysfunction. Sleep-deprived children show the long-lasting effects of sleep loss on a wide range of mental and physical functions, including declined neurocognitive performance, memory loss, anxiety, depression, and obesity [1-6]. These effects seem to be more critical in younger age. For example, longitudinal

\footnotetext{
* Correspondence: mishima@ncnp.go.jp

'Department of Psychophysiology, National Institute of Mental Health, National Center of Neurology and Psychiatry, 4-1-1 Ogawa-Higashi, Kodaira, Tokyo 187-8553, Japan

Full list of author information is available at the end of the article
}

study showed that children with short sleep $(<10 \mathrm{~h})$ before age at 3.5 years were 2.5 -fold more likely to show the externalized problems in age at school entry compared with children with $11 \mathrm{~h}$ of sleep, even if they slept longer later [7].

In daily life, most cases of sleep loss are caused by delayed bedtime (BT). Delayed BT in infants and toddlers is common in developed countries [8,9] including Japan, where the number of such cases has been increasing for some time. A survey of approximately 10,000 preschool children has shown that the rate of children who go to bed at 22:00 or later increased linearly over the period from 1980 to 2000 [10]. Children with delayed BT often exhibit various sleep-related problems, including BT resistance and morning irritability [8,11-14]. Because 
children's sleep-related problems can intensify parent's depressive state and consequently reduce the quality of family functioning [15-18], they are a major public health concern that needs to be urgently addressed.

Even when children regularly have delayed BT, they are generally not allowed to sleep late in the morning so as to fit in with their parents' schedule. Consequently, compared with children with earlier BT, children with delayed BT have shorter total sleep time and longer daytime nap time to compensate for the sleep debt even in toddlers [19]. However, excessive daytime napping diminishes homeostatic sleep drive, and reduced sleep propensity during conventional sleep hours may lead to recurring delays in $\mathrm{BT}$ and persistent sleep debt. A negative cycle between habitually delayed BT, sleep loss, and daytime napping is thought to be a frequent cause of BT resistance, difficulty with morning awakening, and related negative mood and poor appetite [20]. However, the relationships between sleep habits and sleep-related problems at BT and in the morning in children with delayed BT have yet to be elucidated.

To this end, this study aimed to investigate the relationship between delayed BT, sleep habits, and sleeprelated problems at night and in the morning in community-dwelling 2-year-old children.

\section{Methods \\ Participants}

Potential participants were parents/caregivers of 1,570 community-dwelling 2-year-old children born between November 2006 to October 2007 and living in Nishitokyo City, Tokyo (population approximately 197,000). Parents/ caregivers of 829 out of 1,570 children who visited 24month-old health checkup voluntarily were asked to complete a survey on their child's sleep habits and sleeprelated problems. The survey was conducted between November 2008 and October 2009. Children whose age in months, sex, and birth order which were not provided in the survey were excluded from the study.

Written consent was obtained from all participants, who were provided with explanations about the study and its purposes. The study was approved by the Institutional Review Board of the National Center of Neurology and Psychiatry, Japan.

\section{Measures}

To assess children's sleep habits and sleep-related problems, parents/caregivers were asked to answer 32 originally developed questionnaire items and demographical information after receiving an explanation of the study at the checkup. Those who did not have time to fill in the questionnaire at the checkup venue completed the questionnaire at home and returned it by mail.
Sleep habits were evaluated using nine questionnaire items including bed sharing, child's BT, BT irregularity (on average, 90 min per week), sleep onset latency (SOL; minutes to falling asleep at night), number and duration of nocturnal awakenings (WASO), wake time (WT), number of daytime sleeps, and total daytime sleep time (NAP) for the last 1 month. From these items, the following variables were extracted: sleep onset time (SOT; $\mathrm{BT}+\mathrm{SOL}$ ), time in bed (TIB; elapsed interval from BT to WT), total night sleep time (TST; TIB - (SOL + WASO)), and sleep efficiency (SE; ratio of TST to TIB). Answers were excluded if the values were considered highly unlikely or extreme (for example, sleep and wake times appeared to be reversed, total sleep time was less than $4 \mathrm{~h}$, or total sleep time was more than $22 \mathrm{~h}$ ).

Sleep-related problems were evaluated using the remaining 23 items. Part of these items was depicted from the Children's Sleep Habits Questionnaire [21]. Although these items included BT behavior (4 items), behavior occurring during sleep (12 items), problems on morning awakening (5 items), and daytime sleepiness (2 items) in the last 1-month period, 6 items that are thought to be associated with delayed BT were used in the analysis: 'struggles at bedtime', 'wakes up in a negative mood', 'has a hard time getting out of bed', 'takes a long time to be alert', 'has poor appetite in the morning', and 'seems very sleepy.' Each item was evaluated using a 3-point Likert scale (1: rarely [never or once per week], 2: sometimes [two to four times per week], and 3: usually [five or more times per week]). We analyzed these items individually because they were not made as composite items.

\section{Statistical analysis}

One-way analysis of variance (ANOVA) was used for comparisons of sleep habits, except BT irregularity, across the BT groups. Multiple comparisons using Bonferroni's method were then conducted for those variables showing main effects. Chi-square tests were used to compare BT irregularity and the incidence of sleep problems across the BT groups with Bonferroni corrections.

Hierarchical logistic regression was carried out with the incidence of each sleep-related problem (present two or more times per week) as the dependent variable and $\mathrm{BT}$ as the independent variables in model 1 (crude model). In model 2, demographic variables (age in months, sex, birth order) were entered as covariates. In model 3, demographic variables and TST, NAP, and BT irregularity were entered as covariates. BT was divided into four categories based on the clock time, with the category having the earliest BT (before 21:00) regarded as the reference category. Wald's test was used to calculate odd ratios (ORs).

IBM SPSS Statistics version 21.0 (IBM Corporation) was used for statistical analysis, setting $5 \%$ for all critical 
probability levels. Data are expressed as means \pm standard deviation (SD).

\section{Results}

Responses were received from parents/caregivers of 708 children (survey response rate, 85.4\%). As the first 246 of parents/caregivers were asked to answer only sleep habits and 37 out of the remaining 462 children for whom data were incomplete, data were analyzed for 425 children: 204 boys $(48.0 \%), 221$ girls $(52.0 \%)$, mean age 24.1 months (SD, 1.0 month; range, 22 to 30 months), 259 first-borns (60.9\%), 134 second-borns $(31.5 \%)$, and 32 third or later borns (7.5\%).

To reveal associations between delayed BT, sleep habits, and sleep-related problems, children were divided into four groups according to their BT: BT before 21:00 (up to 2100hG; $n=121,28.5 \%$ ), BT between 21:00 and 21:29 (2100hG; $n=137,32.2 \%)$, BT between $21: 30$ and 21:59 (2130hG; $n=77,18.1 \%)$, and BT after 22:00 (2200h or later G; $n=90,21.2 \%)$. No significant differences were seen in mean age $(F(3,421)=0.277, p=0.842)$ between the four groups; however, the male-to-female ratio $\left(\chi^{2}\right.$ $(3)=13.696, p=0.003)$ and the proportion of birth order $\left(\chi^{2}(3)=15.272, \quad p=0.018\right)$ were significantly different among four groups. Boys showed later bedtime than girls, and first-born children showed later bedtime than children with later birth order.

\section{Sleep habits}

Of 425 parent-child units, 423 (99.5\%) shared a bedroom. Mean BT was $21.2(0.9)$ and ranged from 18:00 to 01:00. BT was after 22:00 in 90 children (21.2\%) and was irregular in 178 children (41.9\%). Mean SOL, TST, WT, and NAP were $29.9 \pm 15.7 \mathrm{~min}, 9.4 \pm 0.9 \mathrm{~h}, 7.2 \pm 0.9$ o'clock, and $1.8 \pm 0.7 \mathrm{~h}$, respectively.

Table 1 shows sleep habits in the four different BT groups. WT was delayed significantly, by $1.3 \mathrm{~h}$ on average, with delayed BT from the up to $2100 \mathrm{hG}$ to the
$2200 \mathrm{~h}$ or later $\mathrm{G}(F(3,421)=50.859, p<0.001)$, with a significant difference seen between all groups on multiple comparison analysis (all corrected $p<0.01$ ). With a delayed BT, TIB $(F(3,421)=26.125, p<0.001)$ and TST $(F(3,421)=22.193, p<0.001)$ were significantly decreased, on average by $01.0 \mathrm{~h}$, in the $2200 \mathrm{~h}$ or later $\mathrm{G}$ group compared with the up to $2100 \mathrm{hG}$. The up to $2100 \mathrm{hG}$ had the longest TIB and TST among the groups (all corrected $p<0.01$ ), whereas the $2200 \mathrm{~h}$ or later $\mathrm{G}$ had the shorter TIB and TST compared to the up to $2100 \mathrm{hG}$ and $2100 \mathrm{hG}$ (all corrected $p<0.01$ ). No significant differences in TIB and TST were found between $2130 \mathrm{hG}$ and $2200 \mathrm{~h}$ or later G.

NAP was significantly increased by an average of $0.4 \mathrm{~h}$ with a delayed BT from the up to $2100 \mathrm{hG}$ to the $2200 \mathrm{~h}$ or later $\mathrm{G}(F(3,421)=7.078, p<0.001)$. In addition, the later $\mathrm{BT}$ was, the higher the proportion of children with irregular BT $\left(\chi^{2}(3)=10.163, p=0.017\right)$, and this was the case for $52.2 \%$ of the children in the $2200 \mathrm{~h}$ or later G.

There were no significant differences in $\operatorname{SOL}(F(3,421)=$ $0.266, p=0.850)$, WASO $(F(3,421)=0.247, p=0.864)$, or SE $(F(3,421)=1.040, p=0.375)$ between the four BT groups.

\section{Sleep-related problems}

Table 2 shows sleep-related problems for the four BT groups. Of the six questionnaire items on sleep-related problems, significant differences in prevalence were observed for 'struggles at bedtime' $\left(\chi^{2}(3)=23.017\right.$, corrected $p<0.001)$, 'has a hard time getting out of bed' $\left(x^{2}(3)=21.680, p<0.001\right)$, 'takes a long time to be alert' $\left(X^{2}(3)=12.424, p<0.001\right)$, and 'has poor appetite in the morning' $\left(\chi^{2}(3)=20.516, p=0.001\right)$, but not for 'wakes up in a negative mood' $\left(\chi^{2}(3)=8.513\right.$, corrected $\left.p=0.219\right)$ and 'seems very sleepy' $\left(\chi^{2}(3)=1.841\right.$, corrected $\left.p=1.000\right)$.

Logistic regression analysis of the four items revealed that there were significant associations between BT and sleep-related problems (Table 3). These relationships were observed even after adjusting for TST, NAP, BT

Table 1 Sleep habits by bedtime

\begin{tabular}{|c|c|c|c|c|c|c|c|}
\hline & Whole & Up to $2100 \mathrm{hG}$ & $2100 \mathrm{hG}$ & 2130hG & $2200 \mathrm{~h}$ or later G & $F / X^{2}$ & $p$ \\
\hline Number of participants & 425 & 121 & 137 & 77 & 90 & & \\
\hline Bedtime (BT) (h) & $21.2 \pm 0.9$ & $20.2 \pm 0.5$ & $21.0 \pm 0.1$ & $21.5 \pm 0.1$ & $22.4 \pm 0.6$ & 649.293 & $<0.001$ \\
\hline Sleep onset latency (SOL) (min) & $29.9 \pm 15.7$ & $29.3 \pm 14.4$ & $30.7 \pm 16.6$ & $30.1 \pm 14.7$ & $29.1 \pm 16.7$ & 0.266 & 0.850 \\
\hline Sleep onset time (SOT) (h) & $21.7 \pm 0.9$ & $20.7 \pm 0.5$ & $21.5 \pm 0.3$ & $22.0 \pm 0.2$ & $22.9 \pm 0.6$ & 461.334 & $<0.001$ \\
\hline Wake time (WT) (h) & $7.2 \pm 0.9$ & $6.7 \pm 0.6$ & $7.0 \pm 0.8$ & $7.4 \pm 0.6$ & $7.9 \pm 1.0$ & 50.859 & $<0.001$ \\
\hline Time in bed (TIB) (h) & $10.0 \pm 0.8$ & $10.5 \pm 0.7$ & $10.0 \pm 0.8$ & $9.8 \pm 0.6$ & $9.5 \pm 1.0$ & 26.125 & $<0.001$ \\
\hline Total night sleep time (TST) (h) & $9.4 \pm 0.9$ & $9.9 \pm 0.7$ & $9.4 \pm 1.0$ & $9.2 \pm 0.8$ & $8.9 \pm 1.0$ & 22.193 & $<0.001$ \\
\hline Wake after sleep onset (WASO) (min) & $6.6 \pm 21.4$ & $5.3 \pm 17.3$ & $7.0 \pm 27.1$ & $7.7 \pm 18.1$ & $7.0 \pm 19.6$ & 0.247 & 0.864 \\
\hline Sleep efficiency (SE) (\%) & $93.9 \pm 4.6$ & $94.5 \pm 3.3$ & $93.7 \pm 5.5$ & $93.6 \pm 4.4$ & $93.7 \pm 4.6$ & 1.040 & 0.375 \\
\hline Total daytime sleep time (NAP) (min) & $1.8 \pm 0.7$ & $1.6 \pm 0.5$ & $1.8 \pm 0.8$ & $1.9 \pm 0.6$ & $2.1 \pm 0.6$ & 7.078 & $<0.001$ \\
\hline Bedtime irregularity (\%) & $41.9 \%$ & $33.1 \%$ & $38.7 \%$ & $49.4 \%$ & $52.2 \%$ & 10.163 & 0.017 \\
\hline
\end{tabular}


Table 2 Incidence of sleep-related problems by bedtime

\begin{tabular}{|c|c|c|c|c|c|c|c|c|c|c|c|c|}
\hline & \multicolumn{2}{|c|}{ Whole } & \multicolumn{2}{|c|}{ Up to $2100 \mathrm{hG}$} & \multicolumn{2}{|c|}{ 2100hG } & \multicolumn{2}{|c|}{ 2130hG } & \multicolumn{2}{|c|}{$2200 \mathrm{~h}$ or later G } & \multirow[t]{2}{*}{$x^{2}$} & \multirow[t]{2}{*}{$p$} \\
\hline & $n$ & (\%) & $n$ & (\%) & $n$ & $(\%)$ & $n$ & $(\%)$ & $n$ & (\%) & & \\
\hline Struggles at bedtime & 145 & $(34.1)$ & 25 & $(20.7)$ & 48 & $(35.0)$ & 25 & $(32.5)$ & 47 & $(52.2)$ & 23.017 & $<0.001$ \\
\hline Wakes up in negative mood & 111 & $(26.1)$ & 24 & $(19.8)$ & 31 & $(22.6)$ & 24 & $(31.2)$ & 32 & $(35.6)$ & 8.513 & 0.037 \\
\hline Hard time getting out of bed & 47 & $(11.1)$ & 4 & (3.3) & 11 & $(8.0)$ & 12 & $(15.6)$ & 20 & $(22.2)$ & 21.680 & $<0.001$ \\
\hline Takes long time to be alert & 52 & $(12.2)$ & 6 & $(5.0)$ & 15 & $(10.9)$ & 14 & $(18.2)$ & 17 & $(18.9)$ & 12.424 & $<0.001$ \\
\hline Has poor appetite in the morning & 76 & $(17.9)$ & 11 & $(9.1)$ & 18 & $(13.1)$ & 21 & $(27.3)$ & 26 & $(28.9)$ & 20.516 & $<0.001$ \\
\hline Seems very sleepy & 89 & (20.9) & 26 & $(21.5)$ & 32 & (23.4) & 12 & $(15.6)$ & 19 & $(21.1)$ & 1.841 & 0.606 \\
\hline
\end{tabular}

irregularity, as well as demographic variables (age in months, sex, and birth order). In fully adjusted model (model 3), the ORs between the 2200h or later G and up to $2100 \mathrm{hG}$ groups yielded adjusted ORs of $2.9(95 \%$ CI: 1.5 to $5.6, p=0.002)$ for 'struggles at bedtime', 5.9 (95\% CI: 1.7 to $20.0, p=0.004$ ) for 'has a hard time getting out of bed', 4.1 (95\% CI: 1.4 to $12.0, p=0.010$ ) for 'takes a long time to be alert', and 3.8 (95\% CI: 1.6 to $8.8, p=0.002$ ) for 'has poor appetite in the morning'. Thus, children going to bed 22:00 $\mathrm{h}$ or later was 3 - to 6-fold more likely to experience sleep-related problems than the children going to bed $21: 00 \mathrm{~h}$ or earlier. In addition, compared with the up to $2100 \mathrm{hG}$, the $2100 \mathrm{hG}$ had significant ORs of 1.8 (95\% CI: 1.0 to $3.3, p=0.049$ ) for 'struggles at bedtime' and the $2130 \mathrm{hG}$ had significant ORs of 4.3 (95\% CI: 1.3 to $14.8, p=0.020)$ for 'has a hard time getting out of bed,' 3.9 (95\% CI: 1.4 to 11.3 , $p=0.011$ ) for 'takes a long time to be alert', and 3.6 (95\% CI: 1.5 to $8.4, p=0.003$ ) for 'has poor appetite in the morning'.

\section{Discussion}

This study investigated sleep habits and sleep-related problems in community-dwelling 2-year-old children with delayed BT. As BT was delayed, WT was significantly delayed; however, the magnitude of delay in WT was remarkably smaller than that in BT. As a result, TST was reduced by $1.0 \mathrm{~h}$, from $9.9 \mathrm{~h}$ in the up to $2100 \mathrm{hG}$ to $8.9 \mathrm{~h}$ in the $2200 \mathrm{~h}$ or later G. In contrast, with delayed BT, NAP was increased to compensate for this reduced TST. These results are comparable with previous studies, suggesting that children continuously accumulate sleep debt $[8,11,12]$. In addition, later bedtime was found in boys than girls and first-born children than children with later birth order. One possible factor of sex differences is that boys are vulnerable to the incidence of the developmental disorders, which are greatly likely to show the sleep problems (up to 80\%) [22] including delayed bedtime. Regarding the birth order, Owens et al. [23] showed that children who lived with other children were nearly twice as likely to have early

Table 3 Multivariate logistic regression analysis predicting the incidence of sleep-related problems

\begin{tabular}{|c|c|c|c|c|c|c|c|c|c|c|}
\hline & \multicolumn{2}{|c|}{ Struggles at bedtime } & \multicolumn{2}{|c|}{$\begin{array}{l}\text { Wakes up in } \\
\text { negative mood }\end{array}$} & \multicolumn{2}{|c|}{$\begin{array}{l}\text { Hard time getting } \\
\text { out of bed }\end{array}$} & \multicolumn{2}{|c|}{$\begin{array}{l}\text { Takes a long time } \\
\text { to be alert }\end{array}$} & \multicolumn{2}{|c|}{$\begin{array}{l}\text { Has bad appetite } \\
\text { in the morning }\end{array}$} \\
\hline & OR & $(95 \% \mathrm{Cl})$ & OR & $(95 \% \mathrm{Cl})$ & OR & $(95 \% \mathrm{Cl})$ & OR & $(95 \% \mathrm{Cl})$ & OR & $(95 \% \mathrm{Cl})$ \\
\hline \multicolumn{11}{|c|}{ Model 1 (unadjusted) } \\
\hline $2100 h G$ & 2.1 & $(1.2-3.6)^{*}$ & 1.2 & $(0.6-2.2)$ & 2.6 & $(0.8-8.2)$ & 2.4 & $(0.9-6.3)$ & 1.5 & $(0.7-3.3)$ \\
\hline 2130hG & 1.8 & $(1.0-3.5)$ & 1.8 & $(0.9-3.5)$ & 5.4 & $(1.7-17.4)^{* *}$ & 4.3 & $(1.6-11.6)^{* *}$ & 3.7 & $(1.7-8.3)^{* *}$ \\
\hline 2200 h or later G & 4.2 & $(2.3-7.7)^{* *}$ & 2.2 & $(1.2-4.2)^{*}$ & 8.4 & $(2.7-25.5)^{* *}$ & 4.5 & $(1.7-11.8)^{* *}$ & 4.1 & $(1.9-8.8)^{* *}$ \\
\hline \multicolumn{11}{|c|}{ Model 2 (adjusted for age, gender) } \\
\hline $2100 \mathrm{hG}$ & 2.0 & $(1.1-3.5)^{*}$ & 1.1 & $(0.6-2.0)$ & 2.6 & $(0.8-8.5)$ & 2.3 & $(0.9-6.2)$ & 1.5 & $(0.7-3.3)$ \\
\hline $2130 \mathrm{hG}$ & 1.7 & $(0.9-3.2)$ & 1.7 & $(0.9-3.3)$ & 5.5 & $(1.7-18.0)^{* *}$ & 4.0 & $(1.5-11.1)^{* *}$ & 3.6 & $(1.6-8.1)^{* *}$ \\
\hline 2200h or later G & 3.6 & $(1.9-6.7)^{* *}$ & 2.0 & $(1.0-3.7)^{*}$ & 8.2 & $(2.6-25.4)^{* *}$ & 4.3 & $(1.6-11.6)^{* *}$ & 3.8 & $(1.7-8.4)^{* *}$ \\
\hline \multicolumn{11}{|c|}{ Model 3 (adjusted for age, gender, total sleep time, nap time, bedtime irregularity) } \\
\hline $2100 \mathrm{hG}$ & 1.8 & $(1.0-3.3)^{*}$ & 0.9 & $(0.5-1.7)$ & 2.1 & $(0.6-7.1)$ & 2.2 & $(0.8-6.0)$ & 1.5 & $(0.7-3.4)$ \\
\hline $2130 \mathrm{hG}$ & 1.4 & $(0.7-2.8)$ & 1.4 & $(0.7-2.8)$ & 4.3 & $(1.3-14.8)^{*}$ & 3.9 & $(1.4-11.3)^{*}$ & 3.6 & $(1.5-8.4)^{* *}$ \\
\hline 2200 h or later $G$ & 2.9 & $(1.5-5.6)^{* *}$ & 1.5 & $(0.7-3.0)$ & 5.9 & $(1.7-20.0)^{* *}$ & 4.1 & $(1.4-12.0)^{*}$ & 3.8 & $(1.6-8.8)^{* *}$ \\
\hline
\end{tabular}

OR: odd ratios, $95 \% \mathrm{Cl}: 95 \%$ confidence interval, ${ }^{*} p<0.05,{ }^{* *} p<0.01$. Up to $2100 \mathrm{~h} \mathrm{G}$ was used as the reference category in each model. For a clearer explanation, all of statistically significant results were presented in bold. 
bedtimes compared to those without other children. Thus, increasing the number of family members may stabilize the sleep habits in children.

The study also revealed that sleep loss was not simply associated with earlier BT or earlier sleep onset in 2year-old children with delayed BT. Compared with children with the earliest BT (up to 2100hG), NAP in children with the most delayed BT (2200h or later G) was only $0.5 \mathrm{~h}$ longer even though TST was reduced by $1.0 \mathrm{~h}$. Despite a shorter TST, these children with delayed BT exhibited normal sleep latency and no clear signs of excessive daytime sleepiness (Tables 1 and 2). In adults, daytime arousal can be sustained by afternoon naps lasting only one third of the amount of sleep lost during the night before [24]. Similarly, the present results suggest that also in these children, extended NAP by approximately half of the sleep loss could at least partly compensate for sleep loss and consequently reduced homeostatic sleep pressure for the next night. In accordance with this, Fukuda and Sakashita [20] reported that, compared with kindergarten children with few daytime napping habits, nursery school children with a regular habit of daytime napping often went to bed later, with a high prevalence of sleep onset difficulties. Taken together, the present and previous findings indicate that delayed BT and sleeprelated problems are caught in a negative loop involving reduced sleep pressure due to daytime napping causing BT struggles and delayed BT, and short sleep due to delayed BT resulting in problems in the morning and daytime napping.

On the other hand, children's own biological traits could also contribute to their delayed BTs. Diurnal preferences, chronotypes, regulated by an individual's biological clock largely determines natural sleep and behavioral timings in adults [25-28]. It is reasonable to assume that BTs are self-selected as early as age 2 because a distinct 24-h sleep-wake cycle is formed and sleep is consolidated at night in approximately $90 \%$ of children before the age of 1 year [29] and because approximately $50 \%$ of diurnal preferences are genetically determined [30-32]. The present study also indicates a possible linkage between diurnal preference and sleeprelated problems in children. The logistic regression analysis revealed that delayed BT was significantly correlated with BT resistance and problems in the morning, even after adjusting for TST and NAP (model 3), suggesting that the correlation was not induced solely by insufficient sleep or reduction in sleep pressure due to daytime napping, but could be related to the preference for eveningness. Although the physiological mechanisms between eveningness and sleep problems are less understood, one possible factor is a breakfast composition. Children with poor tryptophan consumption at morning are likely to have difficulties in falling asleep and awakening, and to get angry and depressed [33,34]. Considering that tryptophan is a precursor for serotonin and melatonin, insufficient amount of tryptophan could lead to the malfunctions of circadian and mood regulations.

This study has several limitations. First, children's sleep habits and sleep-related problems were evaluated using questionnaires answered by their parents/caregivers, which has less agreement with objective sleep measurement (that is, actigraphy). Second, as sleep questionnaire used in this study has not been validated, obtained scores do not imply the presence of any kinds of sleep disorders but simply reports the prevalence of sleep problems. Third, we could not provide the parent information such as socioeconomical states and behaviors around children's sleep. Although it is well documented that parental behaviors, especially in mothers [35], could affect to their children's sleep conditions, researches showed sleep interventions for young children have difficulties to improve children's sleep problems [36], suggesting that sleep habits and sleep problems in children are mostly caused by their own characteristics.

Nevertheless, the findings of the present study revealed a large inter-individual variability in BT, TST, and NAP among 2-year-old children. Short sleep was a noticeable feature in children with delayed $\mathrm{BT}$ at a mere 2 years of age, and they also had a high prevalence of BT resistance, difficulty waking up, and other sleep-related problems. To treat children with sleep-related problems, it could be helpful to provide individualized therapy considering both sleep hygiene (daytime napping and BT) and chronobiological predispositions.

\section{Conclusions}

The findings of this study demonstrated that delayed bedtime was associated with various sleep-related problems independent of sleep parameters such as total sleep time and daytime nap time. The causal factors may include diminished homeostatic sleep drive due to prolonged daytime nap as well as diurnal preference (morning or night type) regulated by the biological clock. Taking consideration of individual differences might be useful to improve children's sleep-related problems.

\section{Abbreviations}

BT: Bedtime; SOL: Sleep onset latency; WASO: Nocturnal awakenings; WT: Wake time; NAP: Total daytime sleep time; TIB: Time in bed; TST: Total night sleep time; SE: Sleep efficiency; OR: Odd ratio; Cl: Confidence interval.

\section{Competing interests}

The authors declare that they have no competing interests.

\section{Authors' contributions}

YoK and KM participated in the design of the study. NI, AM, and YoK participated in the data collection. SK, ME, and KM participated in the statistical analysis. SK drafted the manuscript. ME, YuK, NI, AM, YoK, and KM reviewed the manuscript. All authors read and approved the final manuscript. 


\section{Acknowledgements}

This study was supported in part by a Research Grant for Nervous and Mental Disorders (H2O-KOKORO-004) from the Ministry of Health, Labour and Welfare of Japan, and an Intramural Research Grant (No. 23-3) for Neurological and Psychiatric Disorders from the National Center of Neurology and Psychiatry. A part of this study is the result of "Understanding of Molecular and Environmental Bases for Brain Health" carried out under the Strategic Research Program for Brain Sciences by the Ministry of Education, Culture, Sports, Science and Technology of Japan.

\section{Author details}

'Department of Psychophysiology, National Institute of Mental Health, National Center of Neurology and Psychiatry, 4-1-1 Ogawa-Higashi, Kodaira, Tokyo 187-8553, Japan. ${ }^{2}$ Graduate School of Education, The University of Tokyo, 7-3-1 Hongo, Bunkyo-ku, Tokyo 113-8654, Japan. ${ }^{3}$ Support Room for Students with Special Needs, Tokyo Gakugei University, 4-1-1, Nukui Kitamachi, Koganei, Tokyo 184-8501, Japan. ${ }^{4}$ Department of Child and Adolescent Mental Health, National Institute of Mental Health, National Center of Neurology and Psychiatry, 4-1-1 Ogawa-Higashi, Kodaira, Tokyo 187- 8553, Japan.

\section{Received: 17 November 2014 Accepted: 17 February 2015}

\section{Published online: 13 March 2015}

\section{References}

1. Owens J. Classification and epidemiology of childhood sleep disorders. Prim Care. 2008;35(3):533-46. vii.

2. Dewald JF, Meijer AM, Oort FJ, Kerkhof GA, Bogels SM. The influence of sleep quality, sleep duration and sleepiness on school performance in children and adolescents: a meta-analytic review. Sleep Med Rev. 2010;14(3):179-89.

3. Ednick M, Cohen AP, McPhail GL, Beebe D, Simakajornboon N, Amin RS. A review of the effects of sleep during the first year of life on cognitive, psychomotor, and temperament development. Sleep. 2009;32(11):1449-58.

4. Gregory AM, Sadeh A. Sleep, emotional and behavioral difficulties in children and adolescents. Sleep Med Rev. 2012;16(2):129-36.

5. Hoban TF. Sleep disorders in children. Ann N Y Acad Sci. 2010;1184:1-14

6. Moore M. Behavioral sleep problems in children and adolescents. J Clin Psychol Med Settings. 2012;19(1):77-83.

7. Touchette E, Petit D, Seguin JR, Boivin M, Tremblay RE, Montplaisir JY. Associations between sleep duration patterns and behavioral/cognitive functioning at school entry. Sleep. 2007;30(9):1213-9.

8. Mindell JA, Meltzer LJ, Carskadon MA, Chervin RD. Developmental aspects of sleep hygiene: findings from the 2004 National Sleep Foundation Sleep in America Poll. Sleep Med. 2009;10(7):771-9.

9. Mindell JA, Sadeh A, Wiegand B, How TH, Goh DY. Cross-cultural differences in infant and toddler sleep. Sleep Med. 2010;11(3):274-80.

10. Health FIHSCotJSoC. The 2010 infant health survey: short communication. Shoni Hoken Kenkyu. 2011;70(3):448-57. in Japanese.

11. Kohyama J, Shiiki T, Hasegawa T. Sleep duration of young children is affected by nocturnal sleep onset time. Pediatr Int. 2000;42(5):589-91.

12. Kohyama J, Shiiki T, Ohinata-Sugimoto J, Hasegawa T. Potentially harmful sleep habits of 3-year-old children in Japan. J Dev Behav Pediatr. 2002;23(2):67-70.

13. Komada Y, Abe T, Okajima I, Asaoka S, Matsuura N, Usui A, et al. Short sleep duration and irregular bedtime are associated with increased behavioral problems among Japanese preschool-age children. Tohoku J Exp Med. 2011;224(2):127-36.

14. Yokomaku A, Misao K, Omoto F, Yamagishi R, Tanaka K, Takada K, et al. A study of the association between sleep habits and problematic behaviors in preschool children. Chronobiol Int. 2008;25(4):549-64.

15. Hiscock H, Wake M. Randomised controlled trial of behavioural infant sleep intervention to improve infant sleep and maternal mood. BMJ. 2002;324(7345):1062-5.

16. Lam $\mathrm{P}$, Hiscock $H$, Wake M. Outcomes of infant sleep problems: a longitudinal study of sleep, behavior, and maternal well-being. Pediatrics. 2003;111(3):e203-7.

17. Mindell JA, Kuhn B, Lewin DS, Meltzer $\amalg$, Sadeh A. Behavioral treatment of bedtime problems and night wakings in infants and young children. Sleep. 2006;29(10):1263-76.
18. Wake M, Morton-Allen E, Poulakis Z, Hiscock H, Gallagher S, Oberklaid F. Prevalence, stability, and outcomes of cry-fuss and sleep problems in the first 2 years of life: prospective community-based study. Pediatrics. 2006;117(3):836-42.

19. Komada Y, Asaoka S, Abe T, Matsuura N, Kagimura T, Shirakawa S, et al. Relationship between napping pattern and nocturnal sleep among Japanese nursery school children. Sleep Med. 2012;13(1):107-10.

20. Fukuda K, Sakashita Y. Sleeping pattern of kindergartners and nursery school children: function of daytime nap. Percept Mot Skills. 2002;94(1):219-28.

21. Owens JA, Spirito A, McGuinn M. The Children's Sleep Habits Questionnaire (CSHQ): psychometric properties of a survey instrument for school-aged children. Sleep. 2000;23(8):1043-51.

22. Richdale AL, Prior MR. The sleep/wake rhythm in children with autism. Eur Child Adolesc Psychiatry. 1995:4(3):175-86.

23. Owens JA, Jones C, Nash R. Caregivers' knowledge, behavior, and attitudes regarding healthy sleep in young children. J Clin Sleep Med. 2011;7(4):345-50

24. Horne J, Anderson C, Platten C. Sleep extension versus nap or coffee, within the context of 'sleep debt'. J Sleep Res. 2008;17(4):432-6.

25. Hida A, Kitamura S, Enomoto M, Nozaki K, Moriguchi Y, Echizenya M, et al. Individual traits and environmental factors influencing an individual's sleep timing: a study of 225 Japanese couples. Chronobiol Int. 2012;29(2):220-6.

26. Carrier J, Monk TH, Buysse DJ, Kupfer DJ. Sleep and morningness-eveningness in the 'middle' years of life (20-59 y). J Sleep Res. 1997;6(4):230-7.

27. Liu X, Uchiyama M, Shibui K, Kim K, Kudo Y, Tagaya H, et al. Diurnal preference, sleep habits, circadian sleep propensity and melatonin rhythm in healthy human subjects. Neurosci Lett. 2000;280(3):199-202.

28. Taillard J, Philip P, Bioulac B. Morningness/eveningness and the need for sleep. J Sleep Res. 1999:8(4):291-5.

29. Guilleminault C, Anders TF. The pathophysiology of sleep disorders in pediatrics. Part II. Sleep disorders in children. Adv Pediatr. 1976;22:151-74.

30. Barclay NL, Eley TC, Buysse DJ, Archer SN, Gregory AM. Diurnal preference and sleep quality: same genes? A study of young adult twins. Chronobiol Int. 2010;27(2):278-96.

31. Hur YM. Stability of genetic influence on morningness-eveningness: a cross-sectional examination of South Korean twins from preadolescence to young adulthood. J Sleep Res. 2007;16(1):17-23.

32. Koskenvuo M, Hublin C, Partinen M, Heikkila K, Kaprio J. Heritability of diurnal type: a nationwide study of 8753 adult twin pairs. J Sleep Res. 2007;16(2):156-62.

33. Harada T, Hirotani M, Maeda M, Nomura H, Takeuchi H. Correlation between breakfast tryptophan content and morningness-eveningness in Japanese infants and students aged 0-15 yrs. J Physiol Anthropol. 2007;26:201-7.

34. Harada T, Nakade M, Wada K, Akimitsu O, Noji T, Krejci M, et al. Tryptophan and sleep: breakfast tryptophan content and sleep. pp. 473-487 (Chapter 33). In: Preedy VR, Patel VB, Le L-A, editors. Handbook of nutrition, diet and sleep. Wageningen, The Netherlands: Wageningen Academic Publishers; 2013. p. 500.

35. Komada Y, Adachi N, Matsuura N, Mizuno K, Hirose K, Aritomi R, et al. Irregular sleep habits of parents are associated with increased sleep problems and daytime sleepiness of children. Tohoku J Exp Med. 2009;219(2):85-9.

36. Douglas PS, Hill PS. Behavioral sleep interventions in the first six months of life do not improve outcomes for mothers or infants: a systematic review. J Dev Behav Pediatr. 2013;34(7):497-507. 